Check for updates

Cite this: RSC Adv., 2020, 10, 23522

Received 18th March 2020

Accepted 29th May 2020

DOI: $10.1039 / d 0 r a 02525 a$

rsc.li/rsc-advances

\title{
Regio- and diastereoselective synthesis of spiropyrroloquinoxaline grafted indole heterocyclic hybrids and evaluation of their anti- Mycobacterium tuberculosis activity $\uparrow$
}

\author{
Natarajan Arumugam, (DD *a Abdulrahman I. Almansour, ${ }^{\text {* }}$ Raju Suresh Kumar, (D) ${ }^{a}$ \\ Abdul Jaleel Mohammad Ali Al-Aizari, ${ }^{a}$ Shatha Ibrahim Alaqeel, ${ }^{b}$ Sevgi Kansız, (D) \\ Vagolu Siva Krishna, ${ }^{d}$ Dharmarajan Sriram ${ }^{d}$ and Necmi Dege (iD)
}

\begin{abstract}
An efficient and eco compatible approach for the regio- and stereoselective synthesis of structurally diverse novel hybrid heterocycles comprising spiropyrrolidine, indenoquinoxaline and indole structural units in excellent yields, has been achieved through a one-pot multicomponent process involving 1,3-dipolar cycloaddition as a key step. The 1,3-dipolar component is the azomethine ylide generated in situ from indenoquinoxaline and L-tryptophan and reacts with various substituted $\beta$-nitrostyrenes affording the spiroheterocyclic hybrids. The ring system thus created possesses two $\mathrm{C}-\mathrm{C}$ and three $\mathrm{C}-\mathrm{N}$ bonds and four adjacent stereogenic carbons, one of which is quaternary and the reaction proceeded with full diastereomeric control. All the synthesized compounds were assayed for their in vitro activity against Mycobacterium tuberculosis H37Rv using MABA assay. Interestingly, the compound bearing a 2-fluoro substituent on the aryl ring displayed an equipotent activity (MIC $1.56 \mu \mathrm{g} \mathrm{m}^{-1}$ ) to ethambutol against Mycobacterium tuberculosis H37Rv.
\end{abstract}

\section{Introduction}

Tuberculosis (TB) is a chronic infectious disease caused by Mycobacterium tuberculosis bacteria (MTB), that has become an important world-wide public health problem. ${ }^{1}$ According to the World Health Organization (WHO), approximately 10 million people are believed to be infected with TB annually with almost 1.5 million deaths. ${ }^{2}$ Further, HIV-infected patients have an elevated risk of primary or reactivated tuberculosis, and such an active infectious process may enhance HIV replication and increase the risk of death. ${ }^{3}$ It is estimated that about one third of the world's population is infected with latent tuberculosis.

${ }^{a}$ Department of Chemistry, College of Science, King Saud University, P.O Box 2455, Riyadh 11451, Saudi Arabia. E-mail: anatarajan@ksu.edu.sa; aruorgchem@gmail. com; Fax: +966 4675992; Tel: +9664675907

${ }^{b}$ Department of Chemistry, College of Science, King Saud University (034), Riyadh 11495, Saudi Arabia

${ }^{c}$ Department of Fundamental Sciences, Faculty of Engineering, Samsun University, Samsun, 55420, Turkey

${ }^{d}$ Medicinal Chemistry and Antimycobacterial Research Laboratory, Pharmacy Group, Birla Institute of Technology \& Science-Pilani, Hyderabad Campus, Jawahar Nagar, Hyderabad 500078, Telangana, India

${ }^{e}$ Department of Physics, Faculty of Arts and Sciences, Ondokuz Mayls University, Samsun, 55139, Turkey

$\dagger$ Electronic supplementary information (ESI) available. CCDC 1966793. For ESI and crystallographic data in CIF or other electronic format see DOI: 10.1039/d0ra02525a
Currently available first line anti-TB medications viz., ethambutol, isoniazid, pyrazinamide and rifampicin suffer from associated side-effects, poor efficacy in eradicating dormant pathogens and prolonged treatment. ${ }^{4}$ The second line anti-TB drugs such as bedaquiline, delamanid and pretomanid are generally more toxic, less effective and more expensive than the first line anti-TB drugs. Besides, the existence of multidrug and extensively drug resistant tuberculosis (MDR-TB and XDR-TB) further intensifies the problems connected with TB treatment. As patients could become virtually untreatable with the currently available anti-TB drugs, it is imperative to develop structurally diverse, novel, effective, fast acting and affordable anti-TB drugs having a unique mechanism of action with reduced toxicity profiles, capable of overcoming the resistances posed by MDR-TB and XDR-TB to efficiently combat this disease.

In this perspective, spiro compounds are very attractive structural motif for drug discovery since they are intrinsically three dimensional structures that can facilitate interactions with three dimensional binding sites more easily than planar aromatic ring system as ligand. A large number of spiro compounds found in natural products evolved to interact more efficiently with binding pockets in proteins and have better solubility, a crucial property in the process of drug development. Among them, pyrrolidines embedded in a spiro core are prevalent in several alkaloids and synthetic analogs including 


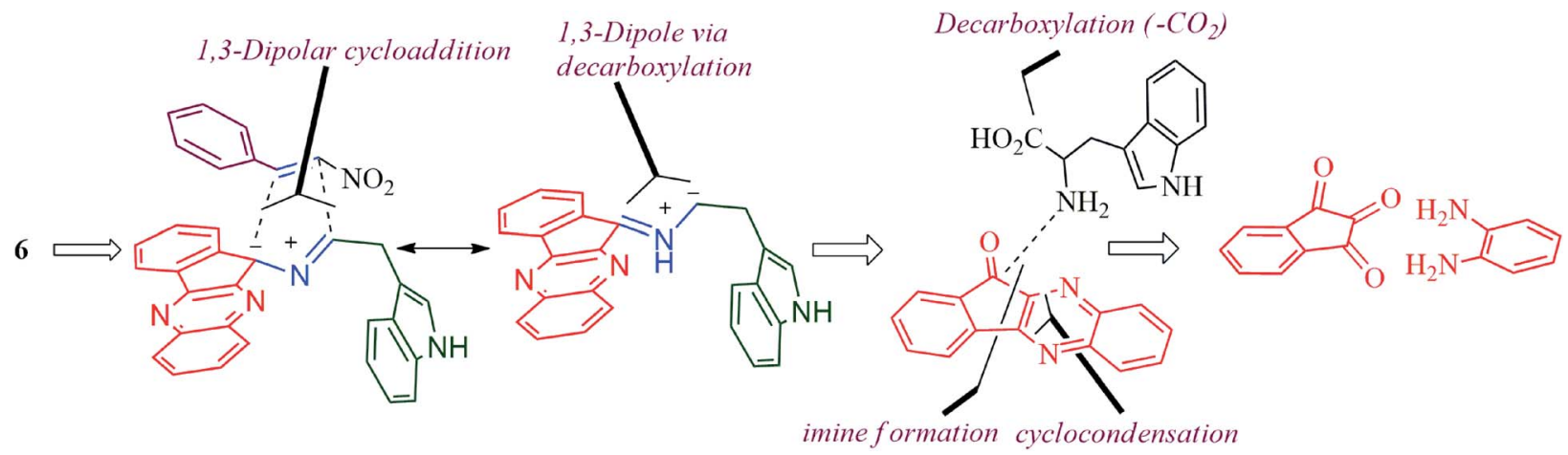

Scheme 1 Synthetic strategy for spiropyrrolo-indenoquinoxaline tethered indole analogs.

spirotryprostatins $\mathrm{A}$ and $\mathrm{B},{ }^{5}$ horsfiline, ${ }^{6}$ elacomine, ${ }^{7}$ formosanine, ${ }^{8}$ rhynchophylline, ${ }^{9}$ MI-219, MI-773 and MI-888. These spiropyrrolidine heterocyclic hybrids displayed interesting biological properties including anticancer, ${ }^{\mathbf{1 0 - 1 2}}$ antimycobacterial, ${ }^{\mathbf{1 3}} \quad$ anti-inflammatory, anti-microbial, ${ }^{\mathbf{1 4}}$ analgesic, ${ }^{15}$ local anesthetic ${ }^{\mathbf{1 6}}$ and AChE inhibition activities. ${ }^{17-19}$ Earlier studies showed that spiropyrrolidine heterocyclic hybrids displayed significant antimycobacterial activities that are comparable or superior to those of some of the currently employed first line TB drugs. ${ }^{20}$

Despite their biological significances, the syntheses of spiropyrrolidine containing indole side chains have received little attention. In the context of our research in the field of 1,3dipolar cycloadditions, ${ }^{21-29}$ herein we report an easy access to the target spiropyrrolo-indenoquinoxaline-indole heterocyclic hybrids via a one-pot, green synthetic protocol employing a 1,3dipolar cycloaddition in ionic liquids. The synthetic strategy planned for the preparation of spiropyrrolo-indenoquinoxaline tethered indole heterocyclic hybrid has been described in Scheme 1. The 1,3-dipole derived from L-tryptophan is relatively less explored in the literature and, to best of our knowledge, this is the first report of the synthesis of 4-nitro-3-phenylspiro [indeno[1,2-b]quinoxaline-pyrrolidin-5-yl)methyl)-indole via one of such a novel class of azomethine ylide, derived from indenoquinoxalinone and L-tryptophan together with their biological evaluation.

\section{Results and discussion}

\subsection{Chemistry}

Our synthetic study begins with a pilot experiment that involved refluxing a mixture of $o$-pheneylenediamine 1 , ninhydrin 2 , Ltryptophan 3 and $\beta$-nitrostyrene $\mathbf{4} \mathbf{k}^{\mathbf{3 0}}$ in methanol for $2 \mathrm{~h}$, which led to the isolation of $3-\left(\left(4^{\prime}\right.\right.$-nitro- $3^{\prime}$-phenylspiro[indeno[1,2- $\left.b\right]$ quinoxaline-11,2'-pyrrolidin]-5'-yl)methyl)- $1 H$-indole $\quad 6 \mathbf{k}$ as a single diastereoisomer in $75 \%$ yield. The same reaction was also examined in different solvents including ethanol, acetonitrile, 1,4-dioxane and methanol :1,4-dioxane (1:1 v/v) under reflux. A slightly improved yield of the product $\mathbf{6 k}$ (77\%) was observed in $\mathrm{MeOH}:$ 1,4-dioxane mixture (Table 1, entry 4). Furthermore, the reaction optimization was also investigated in an ionic liquid [bmim] $\mathrm{Br}$, which led to the formation of the desired product in an excellent yield of $86 \%$ (Table 1, entry 5) in a short reaction time (Scheme 2). Following the optimization study, all subsequent reactions were effected by heating an equimolar mixture of the reactants in [bmim $] \mathrm{Br}$ in an oil bath at $100{ }^{\circ} \mathrm{C}$ for $45 \mathrm{~min}$ and the products 6 were furnished in excellent yields, whilst the ionic liquid could be recovered and reused by simple drying under vacuum. The other possible regioisomer of 6, i.e., compound 7 was not observed even in traces (Scheme 1). It is pertinent to note that the reaction proceeded in a highly regio- and stereoselective fashion generating two new $\mathrm{C}-\mathrm{C}$, three $\mathrm{C}-\mathrm{N}$ bonds and four adjacent stereocenters, one of which is a spiro carbon, in a single operation. It is noteworthy to mention that the rate of the reaction was accelerated by [bmim] $\mathrm{Br}^{31}$ as it possesses electron deficient hydrogen atom which could form hydrogen bonds with heteroatoms of the starting substrates thereby catalyzing the reactions. This multicomponent reaction progressed in a short reaction time with high yield as evidenced by our earlier reports. ${ }^{32-34}$

Structural elucidation of the regioselective spiroheterocyclic hybrids was done carefully using 1D and 2D NMR spectroscopic analysis (vide supporting data) as discussed for a representative example, 6k (Fig. S1, $\uparrow$ vide supporting data). In the ${ }^{1} \mathrm{H}$ NMR spectrum, the $\mathrm{H}-3$ hydrogen appear as a doublet at $\delta 5.04 \mathrm{ppm}(J$ $=10.0 \mathrm{~Hz}$ ) which showed $\mathrm{H}, \mathrm{H}-\mathrm{COSY}$ correlation with the triplet at $\delta 6.75 \mathrm{ppm}(J=9.0 \mathrm{~Hz})$ being assignable to $\mathrm{H}-4$ hydrogen which in turn shows HMBCs (Fig. 1) with the spirocarbons (C-2) and $\mathrm{C}-4$ at $\delta 72.5$ and $\delta 91.0 \mathrm{ppm}$ respectively. The $\mathrm{H}-4$ hydrogen also showed (i) H,H-COSY correlation with a multiplet at $\delta$ 5.13-

Table 1 Solvent optimization for the synthesis of spiroheterocyclic hybrid, 6k

\begin{tabular}{llll}
\hline Entry & Solvents & Time $(\mathrm{h})$ & Yield (\%) \\
\hline 1 & Ethanol & 2 & 72 \\
2 & Methanol & 2 & 75 \\
3 & Acetonitrile & 2 & 69 \\
4 & Methanol $: 1,4$-dioxane & 2 & 77 \\
5 & [bmim]Br & $45 \mathrm{~min}$ & 86
\end{tabular}


<smiles>Nc1ccccc1N</smiles><smiles>O=C1c2ccccc2C(=O)C1(O)O</smiles><smiles>C=C(CC(N)C(=O)O)C1=CC=CCC1</smiles>
$+\mathrm{N}=\mathrm{O}$<smiles>[R]c1ccc(C=C[N+](=O)[O-])cc1</smiles>

\section{3}

$\mathrm{R}=$ a) $\mathrm{H}$, b) $4-\mathrm{Br}$, c) $2-\mathrm{Cl}$, d) $4-\mathrm{Cl}$, e) $4-\mathrm{CH}_{3}$, f) $2 \mathrm{OCH}_{3}$, g) $3-\mathrm{OCH}_{3}$, h) $4-\mathrm{OCH}_{3}$, i) $2-\mathrm{F}$, j) $4-\mathrm{F}$, k) $3-\mathrm{NO}_{2}$
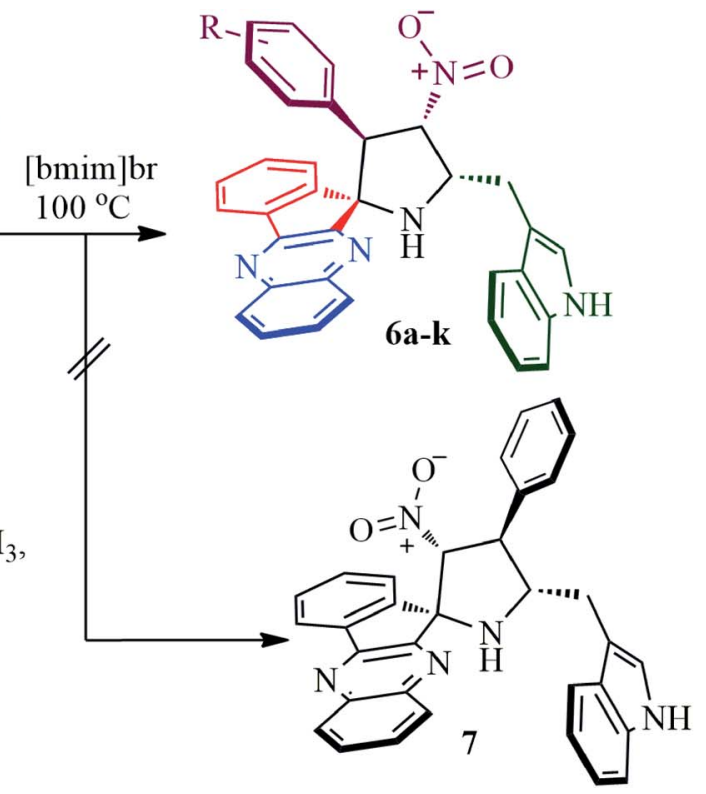

Scheme 2 Synthesis of spiropyrrolo-indenoquinoxaline tethered indole heterocyclic hybrids, $6 a-k$.

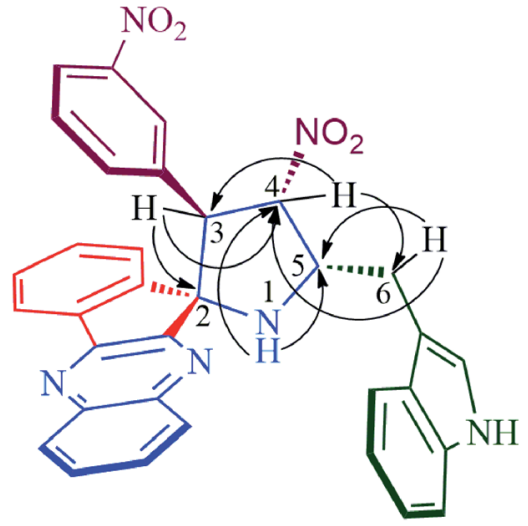

Fig. 1 Selected HMBCs of $6 \mathrm{k}$.

5.19 ppm ascribable to $\mathrm{H}-5$ hydrogen(II) HMBCs with C-3 and C6 at $\delta 57.3$ and $\delta 28.6$ ppm respectively. H-5 hydrogen showed $\mathrm{H}, \mathrm{H}-\mathrm{COSY}$ correlation with the doublet of doublets at $\delta 3.00-$ 3.05 and $\delta 3.09-3.13$ ppm due to H-6 hydrogens. H-6 showed HMBCs with C-5 and C-4 at $\delta 58.5$ and $\delta 91.0$ ppm respectively (vide supporting data). Finally, the regio- and stereochemistry of the spiro cycloadduct was unambiguously ascertained by single crystal X-ray diffraction analysis of $6 \mathrm{e}$ (Fig. 2). ${ }^{35}$

The X-ray diffraction data of 6 e was collected on STOE IPDS 2 (Stoe Imaging Plate Diffraction System II) diffractometer with graphite-monochromatized Mo-K $\alpha$ radiation $(\lambda=0.71073 \AA) .{ }^{36}$ The crystal structure was solved by SHELXT $^{37}$ and refined anisotropically by using SHELXL17/1 (ref. 38) software. WinGX ${ }^{39}$ software was used to prepare material for publication. An additional solvent molecule (2-butanol) of partial occupancy was removed with the SQUEEZE procedure in PLATON. ${ }^{40}$ The asymmetric unit of 6e compound is shown in Fig. 2 and, crystal data, data collection and structure refinement details are summarized in Table $\mathrm{S} 1 \dagger$ (vide supplementary data). Its asymmetric unit contains one independent molecule. The molecular structure is stabilized by the intramolecular $\mathrm{C}-\mathrm{H} \cdots \mathrm{N}$ hydrogen bond (Fig. 2). The crystal packing of 6e features $\mathrm{C}-\mathrm{H} \cdots \mathrm{O}$ and $\mathrm{N}-\mathrm{H} \cdots \mathrm{N}$ hydrogen bonds (Fig. 3 and Table $\mathrm{S} 2, \dagger$ vide supplementary data). The $\mathrm{N}-\mathrm{O}$ bond lengths [1.208 (2) and 1.198 (3) $\mathrm{A}$ ] in the nitro group are close to the values observed for related compounds reported in the literature. ${ }^{\mathbf{4 1 - 4 3}}$ The detailed crystallographic and density functional theory (DFT) studies of spiropyrrolidine will be investigated in due course.

The reaction presumably proceeds through a mechanism proposed in Scheme 3. Thus, the mono ketone 5 initially formed by the reaction of $o$-pheneylenediamine 1 and ninhydrin 2 reacts with L-tryptophan $\mathbf{4}$ to generate the 1,3-dipole 10 via imine 8 and isoxazolidinone intermediate 9. Subsequently, the 1,3-dipole component $\mathbf{1 0}$ attacks the $\beta$-carbon of styrene $\mathbf{4}$ regioselectively to afford the spirocycloadduct 6 (Scheme 2). Furthermore, the spiroheterocycle $\mathbf{6}$ was obtained with complete stereoselectivity. The aryl ring substituted to the pyrrolidine ring adjacent to the spiro carbon being cis to the quinoxaline unit and trans to the nitro group. The transition state (TS1) reveals the unfavorable interaction between the phenyl ring and quinoxaline unit of indenoquinoxaline moiety leading to the unobserved stereoisomer (Scheme 4).

\subsection{Biology}

Compounds $\mathbf{6 a - k}$ thus synthesized were evaluated for their in vitro antimycobacterial activity against Mycobacterium tuberculosis $\mathrm{H} 37 \mathrm{Rv}$ by microplate alamar blue assay (MABA) and test results were presented as minimal inhibition concentration (MIC in $\mu \mathrm{g} \mathrm{mL}^{-1}$ ) as described in Table 2. The following three 


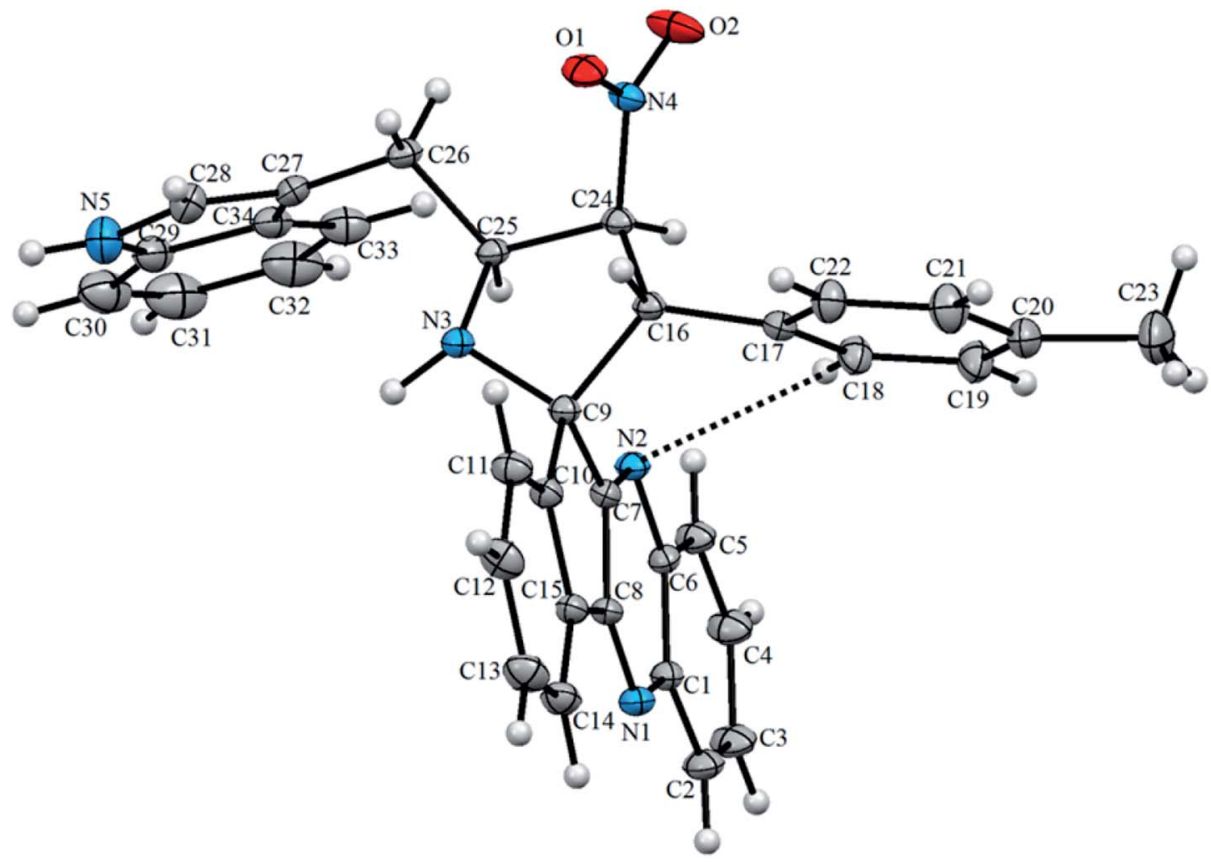

Fig. 2 The asymmetric unit of $6 \mathrm{e}$. Dashed line denotes the intramolecular $\mathrm{C}-\mathrm{H} \cdots \mathrm{N}$ hydrogen bond.

anti-TB leads such as Isoniazid, rifampicin and ethambutol were used as reference standard. Among the synthesized spiropyrrolo-indenoquinoxaline tethered indole heterocyclic hybrids, the four compounds $\mathbf{6 b}, \mathbf{6 c}, \mathbf{6 i}$ and $\mathbf{6 j}$ showed moderate to potent activity which is comparable to that of the standard drug, Ethambutol. Compounds $\mathbf{6 i}$ with $o$-fluoro and $\mathbf{6 j}$ with $p$ - fluoro substituent on the aryl ring possesses remarkable activity against MTB with MIC values 1.56 and $3.125 \mu \mathrm{g} \mathrm{mL} \mathrm{m}^{-1}$, respectively while compounds $\mathbf{6 b}$ and $\mathbf{6 c}$ bearing $p$-bromo and $o$ chloro substituent on the aryl ring displayed moderate activity against MTB with MIC values 6.25 and $12.5 \mu \mathrm{g} \mathrm{mL} \mathrm{m}^{-1}$, respectively. Compound $6 \mathbf{i}$ bearing 2 -fluoro on the aryl ring exhibited

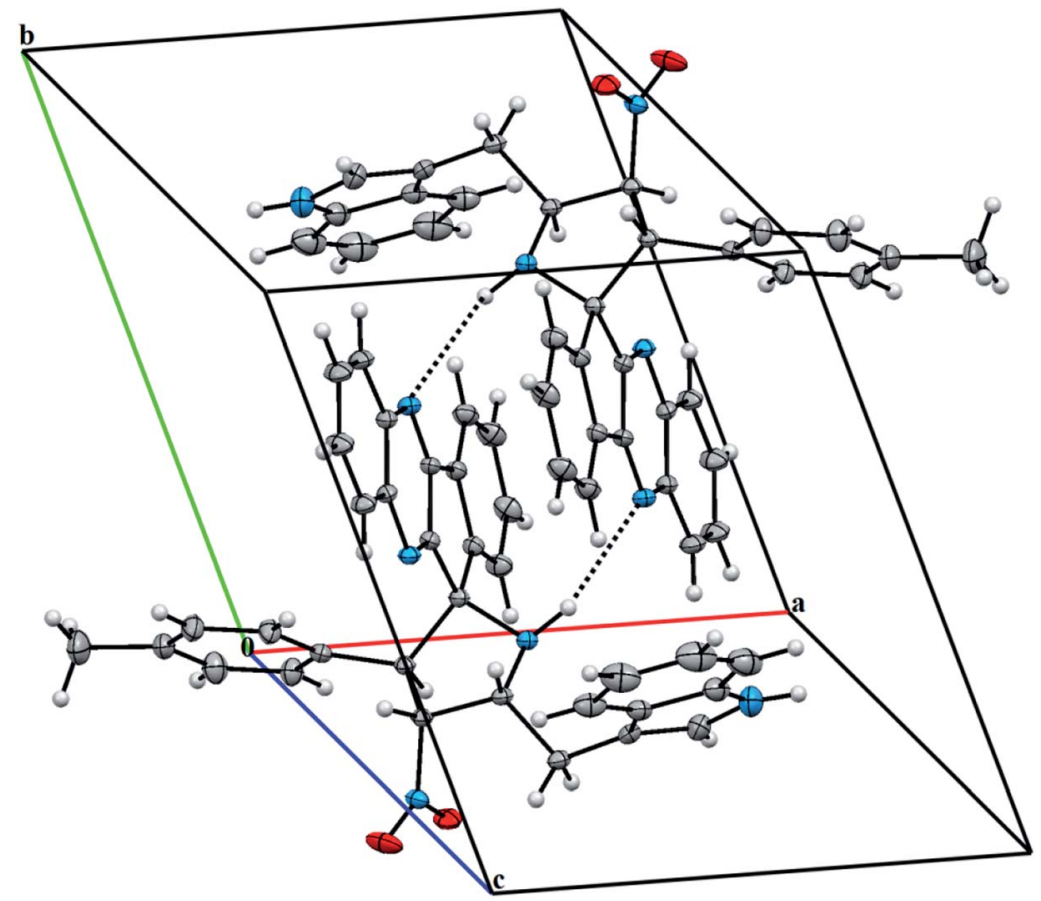

Fig. 3 A partial view of the crystal packing of $6 \mathrm{e}$. Dashed lines denote the intermolecular $\mathrm{N}-\mathrm{H} \cdots \mathrm{N}$ hydrogen bonds. 


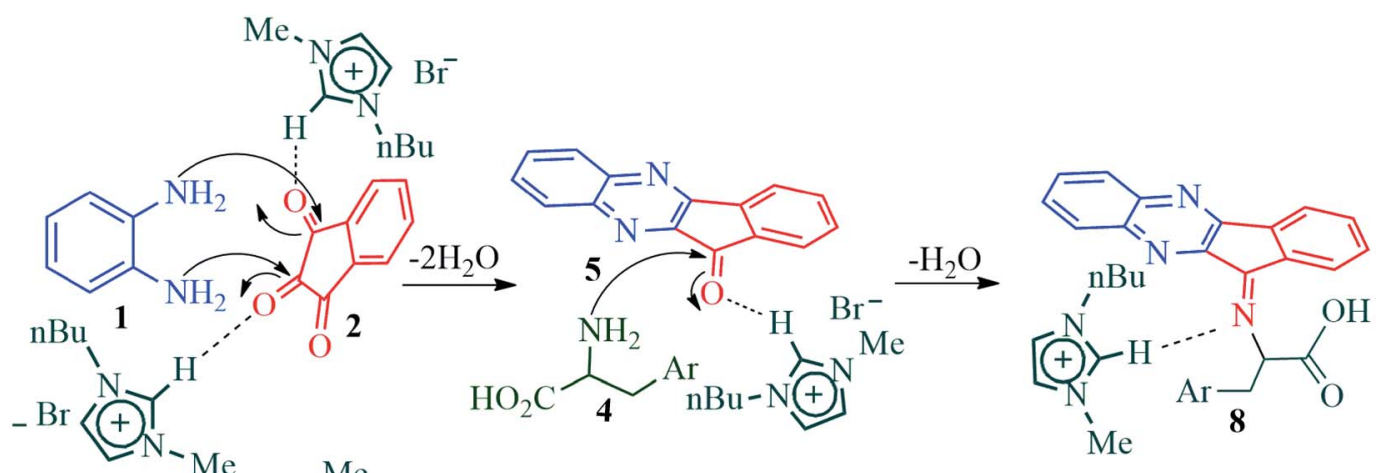

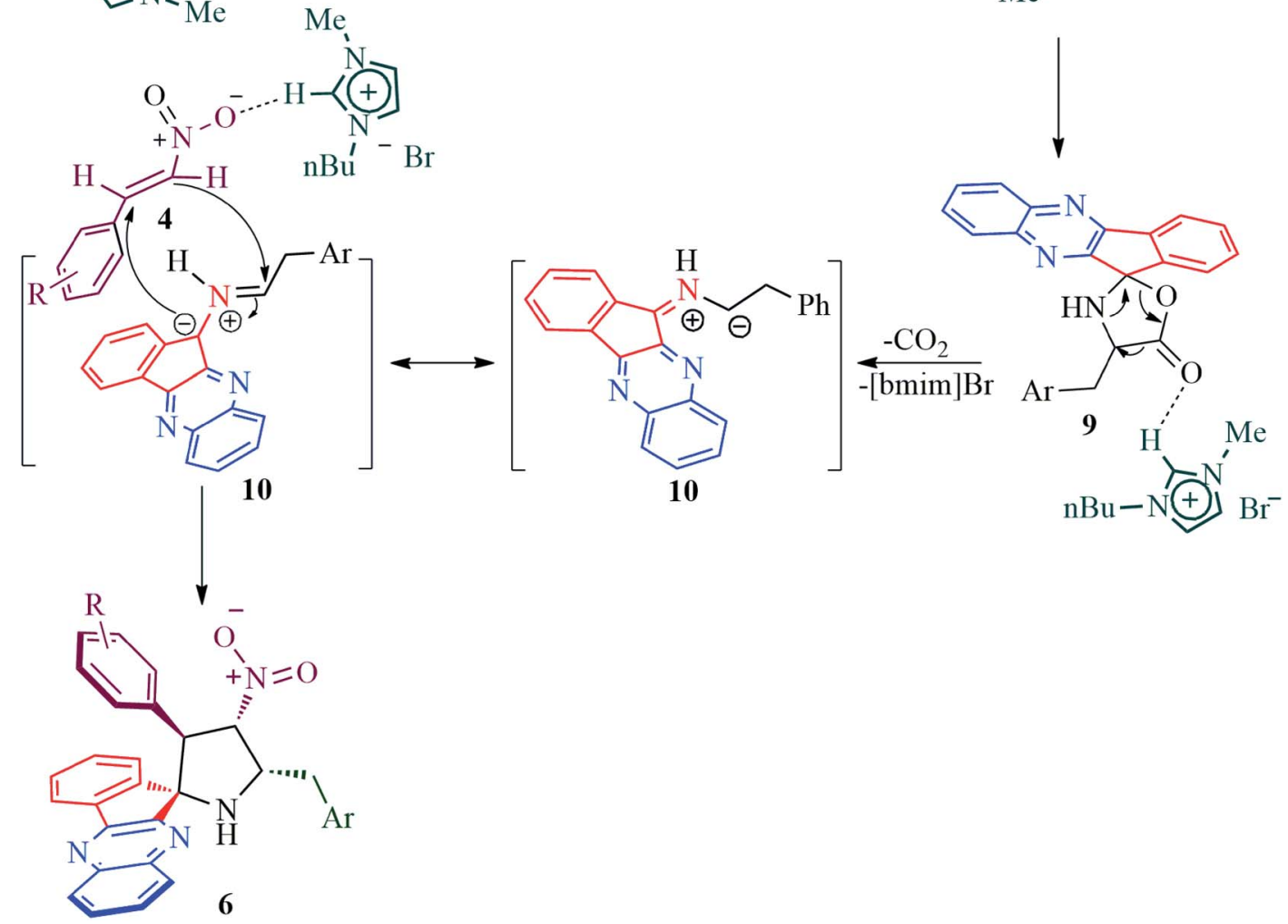

Scheme 3 Plausible mechanism for the formation of observed regiochemistry of spiropyrrolidines, 6 .

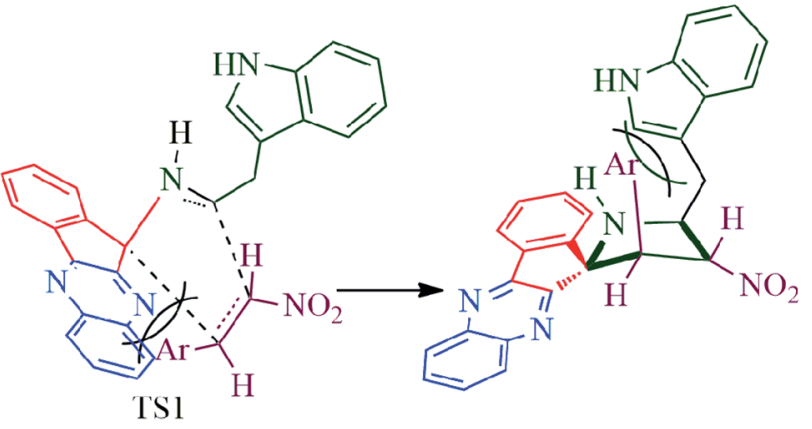

Scheme 4 Explanation of unobserved stereoselectivity.

equipotent activity as ethambutol ( $\mathrm{MIC}=1.56 \mu \mathrm{g} \mathrm{mL} \mathrm{L}^{-1}$ ). The three potent anti-TB compounds viz, $\mathbf{6 b}, \mathbf{6 i}$ and $\mathbf{6 j}$ were tested for their toxicity on normal cell lines. As Mycobacterium tuberculosis resides in macrophages, we have tested them on Raw 264.7 macrophage cell lines and these compounds were found to be safe at $50 \mu \mathrm{g}$ $\mathrm{mL}^{-1}$ test concentration. The percentage of inhibition data of these compounds is summarized in Table 2. The above results revealed that spiropyrrolidines possessing halogen substituents on the phenyl ring displayed significant activity, in particular fluoro substituted spiroheterocyclic hybrids displayed excellent activity.

\section{Conclusion}

The present study describes a regio- and stereoselective synthesis of spiropyrrolo-indenoquinoxaline tethered indole heterocyclic hybrids in excellent yields via [bmim] Br acelerated multicomponent cascade reaction protocol. It is pertinent to note that a new class of non-stabilized 1,3-dipole component generated from L-tryptophan and indenoquinoxalinone was employed. The spiroheterocyclic hybrids possess two $\mathrm{C}-\mathrm{C}$ and three $\mathrm{C}-\mathrm{N}$ bonds and four contiguous stereocenters, out of which one is a spirocarbon. In vitro antimycobacterial activity of 
Table 2 Yield and MIC values and cytotoxicity data of spirpyrrolidine tethered indenoquinoxaline hybrids 6a-k against Mycobacterium tuberculosis

\begin{tabular}{|c|c|c|c|c|}
\hline Entry & Compound & Yield (\%) & $\operatorname{MIC}\left(\mu \mathrm{g} \mathrm{mL}^{-1}\right)$ & $\begin{array}{l}\text { Toxicity (\% inhibition } \\
\text { when tested at } 50 \mu \mathrm{g} \mathrm{mL} L^{-1} \text { ) }\end{array}$ \\
\hline 1 & Ar & 84 & $>25$ & \\
\hline 2 & ;Ar & 81 & 6.25 & 26.15 \\
\hline 3 & & 87 & 12.5 & - \\
\hline 4 & $\Delta r$ & 84 & 25 & - \\
\hline 5 & & 88 & $>25$ & - \\
\hline 6 & & 80 & $>25$ & - \\
\hline 7 & & 79 & $>25$ & - \\
\hline 8 & Ar & 85 & $>25$ & - \\
\hline 9 & & 84 & 1.56 & 32.40 \\
\hline 10 & & 87 & 3.125 & 28.02 \\
\hline 11 & & 81 & $>25$ & - \\
\hline 12 & Isoniazid & & 005 & \\
\hline 13 & Rifampicin & - & $\begin{array}{l}0.05 \\
0.1\end{array}$ & \\
\hline 14 & Ethambutol & - & 1.56 & \\
\hline
\end{tabular}

these synthesized compounds against Mycobacterium tuberculosis $\mathrm{H} 37 \mathrm{Rv}$ revealed that the spiroheterocyclic hybrid bearing $o$ fluoro substituent on the phenyl ring (6i) displayed the most potent activity $\left(1.56 \mu \mathrm{g} \mathrm{mL}^{-1}\right)$ and less toxicity on Raw 264.7 macrophage cell lines at $50 \mu \mathrm{g} \mathrm{mL} \mathrm{m}^{-1}$ concentration suggesting that this compound would be a promising hit for the development of new anti-TB lead compounds. 


\section{Material and methods}

4.1 Synthesis of spiropyrrolo-indenoquinoxaline tethered indole heterocyclic hybrids, 6a-k

An equimolar mixture of aryldiamine 1, triketone 2, L-tryptophan 3 and $\beta$-nitrostyrene 4 in [bmim] Br (200 mg) were heated with stirring at $100{ }^{\circ} \mathrm{C}$ for $45 \mathrm{~min}$. After completion of the reaction (TLC), ethyl acetate $(10 \mathrm{~mL})$ was added and the reaction mixture was further stirred for $10 \mathrm{~min}$. The ethyl acetate layer was separated and the solvent was removed under reduced pressure to afford 6 in excellent yields.

4.1.1 3-(( $4^{\prime}$-Nitro-3' ${ }^{\prime}$-phenylspiro[indeno[1,2-b]quinoxaline11,2' -pyrrolidin]-5'-yl)methyl)-1H-indole, 6a. White solid; Mp: 218-220 ${ }^{\circ} \mathrm{C} ;{ }^{1} \mathrm{H}$ NMR (DMSO-d 6 , $400 \mathrm{MHz}$ ): $\delta / \mathrm{ppm} 3.05-3.10(\mathrm{~m}$, $2 \mathrm{H}), 4.06$ (d, $J=6.0 \mathrm{~Hz}, 1 \mathrm{H}, \mathrm{NH}), 4.87$ (d, $J=10.0 \mathrm{~Hz}, 1 \mathrm{H}), 5.09-$ $5.13(\mathrm{~m}, 1 \mathrm{H}), 6.70-6.78$ (m, 2H), 6.82-6.91 (m, 3H, ArH), 7.007.09 (m, 2H, ArH), 7.25 (d, $J=2.4 \mathrm{~Hz}, 1 \mathrm{H}, \operatorname{ArH}), 7.35$ (d, $J=$ $8.0 \mathrm{~Hz}, 1 \mathrm{H}, \mathrm{ArH}), 7.55(\mathrm{t}, J=8.0 \mathrm{~Hz}, 1 \mathrm{H}, \mathrm{ArH}), 7.70-7.77(\mathrm{~m}, 4 \mathrm{H}$, ArH), 7.84 (d, $J=8.0 \mathrm{~Hz}, 1 \mathrm{H}, \mathrm{ArH}), 7.94-7.96$ (m, 1H, ArH), 8.16$8.22(\mathrm{~m}, 2 \mathrm{H}, \mathrm{ArH}), 10.88$ (s, $1 \mathrm{H}, \mathrm{NH}) ;{ }^{13} \mathrm{C} \mathrm{NMR}\left(\mathrm{CDCl}_{3}, 100\right.$ $\mathrm{MHz}$ ): $\delta / \mathrm{ppm} 28.4,57.3,57.4,71.8,90.5,110.0,111.4,118.3$, 120.9, 123.6, 125.2, 127.3, 127.5, 127.7, 127.9, 128.7, 129.2, 129.3, 129.8, 129.9, 132.3, 132.9, 136.2, 136.7, 140.3, 141.4, 147.5, 153.0, 163.5. LC/MS(ESI): $m / z=523\left(\mathrm{M}^{+}\right)$; anal. calcd for $\mathrm{C}_{33} \mathrm{H}_{25} \mathrm{~N}_{5} \mathrm{O}_{2}$ : C, 75.70; H, 4.81; N, 13.38; found: C, 75.81; $\mathrm{H}$, $4.93 ; \mathrm{N}, 13.49$.

4.1.2 3-((3'-(4-Bromophenyl)-4' -nitrospiro[indeno[1,2-b]quinoxaline-11, $2^{\prime}$-pyrrolidin]-5'-yl)methyl)-1H-indole, $6 \mathbf{b}$. White solid; Mp: $245-247{ }^{\circ} \mathrm{C} ;{ }^{1} \mathrm{H}$ NMR (DMSO-d ${ }_{6}, 400 \mathrm{MHz}$ ): $\delta / \mathrm{ppm}$ 3.02-3.12 (m, 2H), 4.07 (d, $J=6.0 \mathrm{~Hz}, 1 \mathrm{H}, \mathrm{NH}), 4.88$ (d, $J=$ $10.0 \mathrm{~Hz}, 1 \mathrm{H}$ ), 5.07-5.10 (m, 1H), 6.68-6.75 (m, 3H, ArH), 7.00$7.12(\mathrm{~m}, 4 \mathrm{H}, \mathrm{ArH}), 7.24$ (s, 1H, ArH), 7.34 (d, J=8.0 Hz, 1H, ArH), $7.56(\mathrm{t}, J=8.0 \mathrm{~Hz}, 1 \mathrm{H}, \mathrm{ArH}), 7.69-7.79(\mathrm{~m}, 4 \mathrm{H}, \mathrm{ArH}), 7.86(\mathrm{~d}, J=$ $8.0 \mathrm{~Hz}, 1 \mathrm{H}, \mathrm{ArH}), 7.96$ (d, $J=8.0 \mathrm{~Hz}, 1 \mathrm{H}, \mathrm{ArH}), 8.16-8.21(\mathrm{~m}, 2 \mathrm{H}$, $\mathrm{ArH}), 10.87$ (s, $1 \mathrm{H}, \mathrm{NH}) ;{ }^{13} \mathrm{C} \mathrm{NMR}\left(\mathrm{CDCl}_{3}, 100 \mathrm{MHz}\right): \delta / \mathrm{ppm} 28.3$, 56.6, 57.3, 71.7, 90.3, 109.9, 111.4, 118.3, 118.4, 120.9, 121.4, $123.6,125.3,127.3,128.7,129.2$, 129.3, 129.9, 130.0, 130.9, 132.3, 132.4, 136.2, 136.7, 140.2, 141.4, 147.2, 152.9, 163.2. LC/ MS (ESI): $m / z=601\left(\mathrm{M}^{+}\right)$; anal. calcd for $\mathrm{C}_{33} \mathrm{H}_{24} \mathrm{BrN}_{5} \mathrm{O}_{2}: \mathrm{C}, 65.79$; H, 4.02; N, 11.62; found: C, 65.86; H, 4.11; N, 11.73.

4.1.3 3-((3'-(2-Chlorohenyl)-4' -nitrospiro[indeno[1,2-b]quinoxaline-11,2'-pyrrolidin]-5'-yl)methyl)-1H-indole, 6c. White solid; Mp: $228-240{ }^{\circ} \mathrm{C}$; ${ }^{1} \mathrm{H}$ NMR (DMSO-d ${ }_{6}, 400 \mathrm{MHz}$ ): $\delta / \mathrm{ppm}$ 2.92-2.98 (dd, $J=14.0,9.6 \mathrm{~Hz}, 1 \mathrm{H}), 3.04-3.09$ (dd, $J=14.0$, $4.4 \mathrm{~Hz}, 1 \mathrm{H}), 5.26-5.31(\mathrm{~m}, 1 \mathrm{H}), 5.51$ (d, $J=8.0 \mathrm{~Hz}, 1 \mathrm{H}), 6.95(\mathrm{t}, J$ $=9.2 \mathrm{~Hz}, 1 \mathrm{H}), 6.48-6.51(\mathrm{~m}, 1 \mathrm{H}, \mathrm{ArH}), 6.61-6.65(\mathrm{~m}, 1 \mathrm{H}, \mathrm{ArH})$, $6.81(\mathrm{~d}, J=8.4 \mathrm{~Hz}, 1 \mathrm{H}, \mathrm{ArH}), 6.92-6.99$ (m, 4H, ArH), 7.14-7.17 (m, 1H, ArH), 7.24-7.28 (m, 1H, ArH), 7.40-7.44 (m, 1H, ArH), 7.46-7.54 (m, 2H, ArH), 7.61-7.63 (m, 2H, ArH), 7.70-7.73 (m, 1H, ArH), 7.89-7.93 (m, 1H, ArH), 7.99 (d, $J=8.0 \mathrm{~Hz}, 1 \mathrm{H}), 9.47$ $(\mathrm{s}, 1 \mathrm{H}, \mathrm{NH}) ;{ }^{13} \mathrm{C} \mathrm{NMR}\left(\mathrm{CDCl}_{3}, 100 \mathrm{MHz}\right): \delta / \mathrm{ppm} 27.4,53.4,59.1$, 72.0, 93.6, 110.1, 111.1, 118.2, 118.6, 121.2, 123.0, 125.5, 125.7, $126.8,128.3$, 128.5, 128.6, 128.8, 129.0, 129.2, 129.3, 129.5, $129.6,131.1,131.3,134.4,136.1,136.4,140.0$, 141.5, 145.9, 152.8, 162.4. LC/MS(ESI): $m / z=557\left(\mathrm{M}^{+}\right)$; anal. calcd for
$\mathrm{C}_{33} \mathrm{H}_{24} \mathrm{ClN}_{5} \mathrm{O}_{2}$ : C, 71.03; H, 4.34; N, 12.55; found: C, 71.12; H, $4.41 ; \mathrm{N}, 12.63$.

4.1.4 3-((3'-(4-chlorohenyl)-4' -nitrospiro[indeno[1,2-b]quinoxaline-11,2'-pyrrolidin]-5'-yl)methyl)-1H-indole, 6d. White solid; Mp: $212-214{ }^{\circ} \mathrm{C} ;{ }^{1} \mathrm{H}$ NMR (DMSO-d $6,400 \mathrm{MHz}$ ): $\delta / \mathrm{ppm}$ 2.55-2.61 (m, 2H), 4.36 (d, $J=9.6 \mathrm{~Hz}, 1 \mathrm{H}), 4.72-4.82(\mathrm{~m}, 1 \mathrm{H})$, $6.06(\mathrm{t}, J=9.2 \mathrm{~Hz}, 1 \mathrm{H}), 6.23(\mathrm{~d}, J=8.0 \mathrm{~Hz}, 2 \mathrm{H}, \mathrm{ArH}), 6.32(\mathrm{~d}, J=$ 8.4 Hz, 2H, ArH), 6.59-6.62 (m, 2H, ArH), 6.69-6.70 (m, 1H, $\operatorname{ArH}), 6.85$ (d, $J=6.6 \mathrm{~Hz}, 1 \mathrm{H}, \operatorname{ArH}), 7.02$ (t, $J=7.2 \mathrm{~Hz}, 1 \mathrm{H}, \operatorname{ArH})$, $7.22-7.25$ (m, 4H, ArH), 7.34 (d, J=7.6 Hz, 1H, ArH), 7.41 (d, $J=$ $6.4 \mathrm{~Hz}, 1 \mathrm{H}, \mathrm{ArH}), 7.62$ (d, $J=6.4 \mathrm{~Hz}, 2 \mathrm{H}, \mathrm{ArH}), 9.87$ (s, 1H, NH); ${ }^{13} \mathrm{C} \mathrm{NMR}\left(\mathrm{CDCl}_{3}, 100 \mathrm{MHz}\right)$ : $\delta / \mathrm{ppm} 27.2,56.5,56.8,70.9,89.8$, $109.1,110.4$, 117.4, 117.6, 120.2, 123.5, 126.2, 127.0, 127.9, $128.3,128.6,128.7,129.1,130.5,131.2,132.1,135.4,136.0$, 139.5, 140.8, 145.7, 151.8, 157.4, 161.7. LC/MS (ESI): $m / z=557$ $\left(\mathrm{M}^{+}\right)$; anal. Calcd for $\mathrm{C}_{33} \mathrm{H}_{24} \mathrm{ClN}_{5} \mathrm{O}_{2}$ : C, 71.03; H, 4.34; N, 12.55; found: $\mathrm{C}, 71.11 ; \mathrm{H}, 4.43 ; \mathrm{N}, 12.62$.

4.1.5 3-((3'-(4-Methylphenyl)-4' -nitrospiro[indeno[1,2-b]quinoxaline-11, $\mathbf{2}^{\prime}$-pyrrolidin]-5'-yl)methyl)-1H-indole, 6e. White solid; Mp: $186-184{ }^{\circ} \mathrm{C} ;{ }^{1} \mathrm{H}$ NMR (DMSO-d $6,400 \mathrm{MHz}$ ): $\delta / \mathrm{ppm}$ $1.91(\mathrm{~s}, 3 \mathrm{H}), 3.02-3.11(\mathrm{~m}, 2 \mathrm{H}), 4.03$ (d, $J=6.8 \mathrm{~Hz}, 1 \mathrm{H}, \mathrm{NH}), 4.82$ $(\mathrm{d}, J=10.4 \mathrm{~Hz}, 1 \mathrm{H}), 5.07-5.12(\mathrm{~m}, 1 \mathrm{H}), 6.61-6.71(\mathrm{~m}, 5 \mathrm{H}), 7.02-$ 7.09 (m, 2H, ArH), 7.23 (m, 1H, ArH), 7.34 (d, $J=8.0 \mathrm{~Hz}, 1 \mathrm{H}$, $\mathrm{ArH}), 7.54$ (t, $J=7.2 \mathrm{~Hz}, 1 \mathrm{H}, \operatorname{ArH}), 7.54$ (t, $J=7.2 \mathrm{~Hz}, 1 \mathrm{H}, \operatorname{ArH})$, 7.68-7.78 (m, 4H, ArH), 7.84 (d, $J=7.2 \mathrm{~Hz}, 1 \mathrm{H}, \mathrm{ArH}), 7.94-7.97$ (m, 1H, ArH), 8.16-8.19 (m, 1H, ArH), 10.90 (s, 1H, NH); ${ }^{13} \mathrm{C}$ NMR (DMSO-d $6100 \mathrm{MHz}$ ): $\delta /$ ppm 20.2, 28.4, 57.1, 71.8, 90.7, $110.0,118.3,118.4,120.9,121.4,123.6,125.2$, 127.3, 127.6, $128.6,128.7,129.2,129.3,129.7,129.8,132.2$, 136.2, 136.7, 136.8, 140.3, 141.4, 147.6, 153.0, 163.6. LC/MS(ESI): $m / z=537$ $\left(\mathrm{M}^{+}\right)$; anal. calcd for $\mathrm{C}_{34} \mathrm{H}_{27} \mathrm{~N}_{5} \mathrm{O}_{2}$ : C, 75.96; H, 5.06; N, 13.03; found: $\mathrm{C}, 76.05 ; \mathrm{H}, 5.18 ; \mathrm{N}, 13.12$.

4.1.6 3-((3'-(2-Methoxyphenyl)-4' $\mathbf{4}^{\prime}$-nitrospiro[indeno[ $[1,2-b]$ quinoxaline-11, $\mathbf{2}^{\prime}$-pyrrolidin]-5'-yl)methyl)-1H-indole, 6f. White solid; Mp: $199-201{ }^{\circ} \mathrm{C} ;{ }^{1} \mathrm{H}$ NMR (DMSO-d $\left.{ }_{6}, 400 \mathrm{MHz}\right): \delta / \mathrm{ppm}$ 3.03-3.13 (m, 2H), 3.21 (s, 3H), 4.03 (d, $J=5.6 \mathrm{~Hz}, 1 \mathrm{H}, \mathrm{NH})$, $5.21-5.23(\mathrm{~m}, 1 \mathrm{H}), 5.45(\mathrm{~d}, J=8.8 \mathrm{~Hz}, 1 \mathrm{H}), 6.35(\mathrm{t}, J=7.6 \mathrm{~Hz}$, 1H), 6.54-6.60 (m, 2H, ArH), 6.83 (t, J=8.0 Hz, 1H, ArH) 6.92 (d, $J=7.2 \mathrm{~Hz}, 1 \mathrm{H}, \mathrm{ArH}), 7.02-7.08$ (m, 2H, ArH), 7.25 (m, 1H, ArH), 7.33-7.35 (m, 1H, ArH), 7.51 (t, $J=7.2 \mathrm{~Hz}, 1 \mathrm{H}, \mathrm{ArH}), 6.71-6.77$ (m, 4H, ArH), 7.82 (d, $J=7.2 \mathrm{~Hz}, 1 \mathrm{H}, \mathrm{ArH}), 7.94$ (d, $J=7.2 \mathrm{~Hz}$, 1H, ArH), 7.14-7.16 (m, 2H, ArH), 10.86 (s, 1H, NH); ${ }^{13} \mathrm{C}$ NMR (DMSO-d 6 , $100 \mathrm{MHz}$ ): $\delta / \mathrm{ppm} \mathrm{28.1,} \mathrm{50.3,} \mathrm{54.8,} \mathrm{58.2,} \mathrm{71.8,} \mathrm{91.7,}$ $110.1,110.8,111.4,118.3,119.5,120.9,121.3,123.6,126.0$, $127.3,128.1$, 128.5, 128.6, 129.1, 129.2, 129.4, 129.6, 131.5, 136.3, 140.1, 141.3, 148.1, 153.1, 157.1, 164.0. LC/MS (ESI): $m / z$ $=553\left(\mathrm{M}^{+}\right)$; anal. calcd for $\mathrm{C}_{34} \mathrm{H}_{27} \mathrm{~N}_{5} \mathrm{O}_{3}: \mathrm{C}, 73.76 ; \mathrm{H}, 4.92 ; \mathrm{N}$, 12.65; found: $\mathrm{C}, 73.87 ; \mathrm{H}, 4.85 ; \mathrm{N}, 12.54$.

4.1.7 3-((3'-(3-Methoxyphenyl)-4' -nitrospiro[indeno[1,2-b]quinoxaline-11, $2^{\prime}$-pyrrolidin]-5'-yl)methyl)-1 $H$-indole, $\quad 6 \mathrm{~g}$. White solid; Mp: $276-278{ }^{\circ} \mathrm{C} ;{ }^{1} \mathrm{H}$ NMR (DMSO-d 6 , $400 \mathrm{MHz}$ ): $\delta /$ ppm 3.08-3.15 (m, 2H), 3.23 (s, 3H), 4.07 (d, $J=6.0 \mathrm{~Hz}, 1 \mathrm{H}$, $\mathrm{NH}), 4.86(\mathrm{~d}, J=10.4 \mathrm{~Hz}, 1 \mathrm{H}), 5.11-5.15(\mathrm{~m}, 1 \mathrm{H}), 6.29(\mathrm{~m}, 1 \mathrm{H})$, $6.38(\mathrm{~d}, J=8.0 \mathrm{~Hz}, 1 \mathrm{H}, \mathrm{ArH}), 6.46$ (d, $J=8.0 \mathrm{~Hz}, 1 \mathrm{H}, \mathrm{ArH}), 6.69-$ 6.79 (m, 2H, ArH), 7.02-7.10 (m, 2H, ArH), 7.26 (m, 1H, ArH), $7.36(\mathrm{~d}, J=8.0 \mathrm{~Hz}, 1 \mathrm{H}, \operatorname{ArH}), 7.55(\mathrm{t}, J=7.2 \mathrm{~Hz}, 1 \mathrm{H}, \operatorname{ArH})$, 7.68-7.77 (m, 4H, ArH), 7.85 (d, $J=8.0 \mathrm{~Hz}, 1 \mathrm{H}, \mathrm{ArH}), 7.97$ (d, $J$ 
$=7.6 \mathrm{~Hz}, 1 \mathrm{H}, \mathrm{ArH}), 8.17-8.21(\mathrm{~m}, 2 \mathrm{H}, \mathrm{ArH}), 10.87(\mathrm{~s}, 1 \mathrm{H}, \mathrm{NH})$; ${ }^{13} \mathrm{C}$ NMR (DMSO-d 6 , $100 \mathrm{MHz}$ ): $\delta / \mathrm{ppm} \mathrm{28.4,} \mathrm{50.5,} \mathrm{54.2,} \mathrm{57.6,}$ 71.9, 90.7, 110.0, 111.3, 111.5, 113.0, 113.4, 118.5, 120.9, 121.2, $121.4,123.8,123.9,125.2,127.3,129.2,129.6,129.9,132.5$, 134.4, 136.3, 136.8, 140.3, 141.4, 147.6, 153.0, 158.5, 163.6. LC/ MS (ESI): $m / z=553\left(\mathrm{M}^{+}\right)$; anal. calcd for $\mathrm{C}_{34} \mathrm{H}_{27} \mathrm{~N}_{5} \mathrm{O}_{3}: \mathrm{C}, 73.76$; $\mathrm{H}, 4.92 ; \mathrm{N}, 12.65$; found: $\mathrm{C}, 73.84 ; \mathrm{H}, 4.98 ; \mathrm{N}, 12.71$.

4.1.8 3-((3'-(4-Methoxyphenyl)-4' ${ }^{\prime}$-nitrospiro[indeno[1,2-b]quinoxaline-11,2'-pyrrolidin]-5'-yl)methyl)-1H-indole, $6 \mathrm{~h}$. White solid; Mp: $262-264{ }^{\circ} \mathrm{C}$; ${ }^{1} \mathrm{H}$ NMR (DMSO-d 6 , $400 \mathrm{MHz}$ ): $\delta / \mathrm{ppm} 3.02-3.11(\mathrm{~m}, 2 \mathrm{H}), 3.41(\mathrm{~s}, 3 \mathrm{H}), 4.03(\mathrm{~d}, J=6.4 \mathrm{~Hz}, 1 \mathrm{H}$, $\mathrm{NH}), 4.81(\mathrm{~d}, J=10.0 \mathrm{~Hz}, 1 \mathrm{H}), 5.05-5.10(\mathrm{~m}, 1 \mathrm{H}), 6.39(\mathrm{~m}, 2 \mathrm{H}$, $\operatorname{ArH}), 6.49(\mathrm{t}, J=9.6 \mathrm{~Hz}, 1 \mathrm{H}, \operatorname{ArH}), 6.67-6.70(\mathrm{~m}, 2 \mathrm{H}, \operatorname{ArH})$, 7.00-7.09 (m, 2H, ArH), 7.23-7.24 (m, 1H, ArH), $7.34(\mathrm{~d}, J=$ $8.0 \mathrm{~Hz}, 1 \mathrm{H}, \mathrm{ArH}), 7.54(\mathrm{t}, J=8.0 \mathrm{~Hz}, 1 \mathrm{H}, \mathrm{ArH}), 7.68-7.78(\mathrm{~m}, 4 \mathrm{H}$, $\operatorname{ArH}), 7.85$ (d, $J=8.0 \mathrm{~Hz}, 1 \mathrm{H}, \operatorname{ArH}), 7.97$ (d, $J=7.6 \mathrm{~Hz}, 1 \mathrm{H}, \operatorname{ArH})$, 8.17-8.20 (m, 2H, ArH), 10.87 (s, $1 \mathrm{H}, \mathrm{NH}) ;{ }^{13} \mathrm{C}$ NMR (DMSO-d 6 , $100 \mathrm{MHz}$ ): $\delta / \mathrm{ppm} 28.4,54.7,56.8,57.0,71.8,90.9,110.0,111.4$, $113.4,118.3,120.9,121.4,123.6,124.5,125.2$, 127.3, 128.7, $128.9,129.2,129.3,129.7,129.8,132.2,136.2,136.7,140.3$, 141.4, 147.6, 153.1, 158.4, 163.7. LC/MS (ESI): $m / z=553\left(\mathrm{M}^{+}\right)$; anal. calcd for $\mathrm{C}_{34} \mathrm{H}_{27} \mathrm{~N}_{5} \mathrm{O}_{3}$ : C, 73.76; H, 4.92; N, 12.65; found: C, 73.88; H, 4.84; N, 12.76 .

4.1.9 3-(( $3^{\prime}-(2-$ Fluorophenyl)-4' - nitrospiro[indeno[1,2-b]quinoxaline-11, $\mathbf{2}^{\prime}$-pyrrolidin]-5'-yl)methyl)-1 $\boldsymbol{H}$-indole, 6i. White solid; Mp: $175-177^{\circ} \mathrm{C} ;{ }^{1} \mathrm{H}$ NMR (DMSO-d 6 , $400 \mathrm{MHz}$ ): $\delta / \mathrm{ppm} 3.01-$ 3.07 (dd, $J=13.6,8.8 \mathrm{~Hz}, 1 \mathrm{H}), 3.11-3.20$ (m, 1H), 4.11 (d, $J=$ $6.4 \mathrm{~Hz}, 1 \mathrm{H}, \mathrm{NH}), 5.18-5.22(\mathrm{~m}, 1 \mathrm{H}), 5.28$ (d, J=9.6 Hz, 1H), 6.61$6.68(\mathrm{~m}, 2 \mathrm{H}, \mathrm{ArH}), 6.99(\mathrm{t}, J=9.6 \mathrm{~Hz}, 1 \mathrm{H}, \mathrm{ArH}), 6.91-6.94(\mathrm{~m}, 1 \mathrm{H}$, ArH), 7.01-7.12 (m, 3H, ArH), 7.26-7.27 (m, 1H, ArH), 7.34 (d, $J=$ $8.0 \mathrm{~Hz}, 1 \mathrm{H}, \mathrm{ArH}), 7.53$ (t, $J=8.0 \mathrm{~Hz}, 1 \mathrm{H}, \mathrm{ArH}), 7.71-7.78(\mathrm{~m}, 3 \mathrm{H}$, ArH), 7.83 (d, $J=8.0 \mathrm{~Hz}, 1 \mathrm{H}, \operatorname{ArH}), 7.96$ (d, $J=7.6 \mathrm{~Hz}, 1 \mathrm{H}, \operatorname{ArH}$ ), 8.13-8.19 (m, 3H, ArH); ${ }^{13} \mathrm{C}$ NMR (DMSO-d $6,100 \mathrm{MHz}$ ): $\delta / \mathrm{ppm}$ 28.2 , 58.3, 63.9, 71.7, 91.2, 109.9, 110.7, 111.2, 118.3, 118.5, 120.1, 120.2, 121.0, 121.2, 123.8, 123.6, 125.5, 127.3, 129.3, 129.5, 129.7, $132.3,136.3,136.4,136.6,140.2,141.5,147.2,153.0,159.0,160.3(J$ $=246.2 \mathrm{~Hz}), 163.4$. LC/MS (ESI): $m / z=553\left(\mathrm{M}^{+}\right)$; anal. calcd for $\mathrm{C}_{33} \mathrm{H}_{24} \mathrm{FN}_{5} \mathrm{O}_{2}$ : C, 73.19; $\mathrm{H}, 4.47 ; \mathrm{N}, 12.93$; found: C, 73.30; H, $4.60 ; \mathrm{N}, 13.02$.

4.1.10 3-((3'-(4-Fluorophenyl)-4' -nitrospiro[indeno[1,2-b]quinoxaline-11, $2^{\prime}$-pyrrolidin]-5'-yl)methyl)-1 $H$-indole, $6 j$. White solid; Mp: $168-170{ }^{\circ} \mathrm{C} ;{ }^{1} \mathrm{H}$ NMR (DMSO-d $\left.{ }_{6}, 400 \mathrm{MHz}\right): \delta /$ ppm 3.02-3.13 (m, 2H), 4.06 (d, $J=6.6 \mathrm{~Hz}, 1 \mathrm{H}, \mathrm{NH}), 4.99$ (d, $J$ $=9.6 \mathrm{~Hz}, 1 \mathrm{H}), 5.09-5.13(\mathrm{~m}, 1 \mathrm{H}), 6.67-6.72(\mathrm{~m}, 3 \mathrm{H}, \mathrm{ArH})$, 6.81-6.84 (m, 2H, ArH), 7.00-7.09 (m, 2H, ArH), 7.24-7.25 (m, $1 \mathrm{H}, \mathrm{ArH}), 7.35$ (d, $J=7.2 \mathrm{~Hz}, 1 \mathrm{H}, \operatorname{ArH}), 7.56(\mathrm{t}, J=8.0 \mathrm{~Hz}, 1 \mathrm{H}$, ArH), 7.69-7.77 (m, 4H, ArH), 7.86 (d, $J=8.0 \mathrm{~Hz}, 1 \mathrm{H}, \operatorname{ArH})$, $7.97(\mathrm{~d}, J=7.6 \mathrm{~Hz}, 1 \mathrm{H}, \mathrm{ArH}), 8.16-8.22$ (m, 2H, ArH); ${ }^{13} \mathrm{C} \mathrm{NMR}$ (DMSO-d ${ }_{6}, 100 \mathrm{MHz}$ ): $\delta / \mathrm{ppm} 28.3,56.6,57.3$, 71.8, 90.6, 109.9, 111.4 , 114.7, 114.9, 118.3, 118.4, 120.9, 121.5, 123.6, 125.3, $127.3,128.7,129.2$, 129.7, 129.8, 129.9, 130.0, 132.3, 136.2, 136.7, 140.3, 141.4, 147.3, 153.0, $161.3(\mathrm{~J}=243.0 \mathrm{~Hz}), 163.4$. LC/MS (ESI): $m / z=553\left(\mathrm{M}^{+}\right)$; anal. calcd for $\mathrm{C}_{33} \mathrm{H}_{24} \mathrm{FN}_{5} \mathrm{O}_{2}: \mathrm{C}$, 73.19; H, 4.47; N, 12.93; found: C, 73.28; H, 4.56; N, 13.01.

4.1.11 3-((3'-(3-Nitrophenyl)-4' -nitrospiro[indeno[1,2-b]quinoxaline-11, $\mathbf{2}^{\prime}$-pyrrolidin]-5' -yl)methyl)-1H-indole, 6k. White solid; Mp: $254-256{ }^{\circ} \mathrm{C} ;{ }^{1} \mathrm{H}$ NMR (DMSO-d ${ }_{6}, 500 \mathrm{MHz}$ ): $\delta / \mathrm{ppm}$
3.00-3.05 (dd, $J=14.0,9.0 \mathrm{~Hz}, 1 \mathrm{H}), 3.09-3.13(\mathrm{dd}, J=14.5$, $4.5 \mathrm{~Hz}, 1 \mathrm{H}), 4.10(\mathrm{~d}, J=6.0 \mathrm{~Hz}, 1 \mathrm{H}, \mathrm{NH}), 5.04(\mathrm{~d}, J=10.0 \mathrm{~Hz}$, $1 \mathrm{H}), 5.13-5.19(\mathrm{~m}, 1 \mathrm{H}), 6.75(\mathrm{t}, J=9.0 \mathrm{~Hz}, 1 \mathrm{H}), 6.99-7.06(\mathrm{~m}, 2 \mathrm{H}$, $\operatorname{ArH}), 7.11$ (t, $J=8.0 \mathrm{~Hz}, 1 \mathrm{H}, \operatorname{ArH}), 7.23-7.26$ (m, 2H, ArH), 7.32 (d, $J=8.0 \mathrm{~Hz}, 1 \mathrm{H}, \mathrm{ArH}), 7.54$ (t, $J=7.0 \mathrm{~Hz}, 1 \mathrm{H}, \mathrm{ArH}), 7.64-7.65$ (m, 1H, ArH), 7.69-7.72 (m, 3H, ArH), 7.73-7.78 (m, 2H, ArH), 7.81 (d, $J=7.5 \mathrm{~Hz}, 1 \mathrm{H}, \mathrm{ArH}), 7.88-7.90$ (m, 1H, ArH), 8.15-8.17 $(\mathrm{m}, 1 \mathrm{H}, \mathrm{ArH}), 8.24$ (d, J=7.5 Hz, 1H, ArH) $10.84(\mathrm{~s}, 1 \mathrm{H}, \mathrm{NH}) ;{ }^{13} \mathrm{C}$ NMR (DMSO-d 6 , $125 \mathrm{MHz}$ ): $\delta /$ ppm 28.6, 57.3, 58.5, 72.5, 91.0, 110.5 , 111.9, 118.9, 119.0, 121.5, 122.0, 123.0, 123.1, 124.2, $125.9,127.9,129.2,129.8,129.9,130.0,130.5,130.7,133.0$, 135.1 , 136.0, 136.8, 137.2, 140.8, 141.9, 147.4, 147.5, 153.3, 163.5. LC/MS (ESI): $m / z=568\left(\mathrm{M}^{+}\right)$; anal. calcd for $\mathrm{C}_{33} \mathrm{H}_{24} \mathrm{~N}_{6} \mathrm{O}_{4}$ : C, 69.71; H, 4.25; N, 14.78; found: C, 69.80; H, 4.33; N, 14.85.

\section{Conflicts of interest}

There are no conflicts to declare.

\section{Acknowledgements}

The authors acknowledge the Deanship of Scientific Research at King Saud University for funding this work through the Research grant RG-1440-071.

\section{References}

1 N. Fogel, a disease without boundaries, Tuberculosis, 2015, 95, 527.

2 World Health Organization, Global Tuberculosis Report, 2019, http://www.who.int/tb/publications/global_report/en/.

3 K. M. De Cock and R. E. Chaisson, A reappraisal of tuberculosis control in countries with high rates of HIV infection, Int. J. Tubercul. Lung Dis., 1999, 3, 457-465.

4 A. Somoskovi, L. M. Parson and M. Salfinger, The Molecular Basis of Resistance to Isoniazid, Rifampin, and Pyrazinamide in Mycobacterium tuberculosis, Respir. Res., 2001, 2, 164-168.

5 G. Bhaskar, Y. Arun, C. Balachandran, C. Saikumar and P. T. Perumal, Synthesis of novel spirooxindole derivatives by one pot multicomponent reaction and their antimicrobial activity, Eur. J. Med. Chem., 2012, 51, 79-91.

6 S. N. Singha, S. Regati, A. K. Paul, M. Layeka, S. Jayaprakasha, K. V. Reddy, G. S. Deora, S. Mukherjeee and M. Pal, Cumediated 1,3-dipolar cycloaddition of azomethine ylides with dipolarophiles: a faster access to spirooxindoles of potential pharmacological interest, Tetrahedron Lett., 2013, 54, 5448-5452.

7 N. Deppermann, H. Thomanek, A. H. G. P. Prenzel and W. Maison, Pd-Catalyzed Assembly of Spirooxindole Natural Products: A Short Synthesis of Horsfiline, J. Org. Chem., 2010, 75, 5994-6000.

8 S. Lanka, S. Thennarasu and P. T. Perumal, Facile synthesis of novel dispiroheterocylic derivatives through cycloaddition of azomethine ylides with acenaphthenone-2-ylidine ketones, Tetrahedron Lett., 2012, 53, 7052-7055. 
9 A. Pesquet and M. Othman, Combining $\alpha$-amidoalkylation reactions of $\mathrm{N}$-acyliminium ions with ring-closing metathesis: access to versatile novel isoindolones spirocyclic compounds, Tetrahedron Lett., 2013, 54, 52275231.

10 B. Yu, D. Q. Yu and H. M. Liu, Spirooxindoles: Promising scaffolds for anticancer agents, Eur. J. Med. Chem., 2015, 97, 673-698.

11 D. Kathirvelan, J. Haribabu, B. S. R. Reddy, C. Balachandran and V. Duraipandiyan, Facile and diastereoselective synthesis of $3,2^{\prime}$-spiropyrrolidine-oxindoles derivatives, their molecular docking and antiproliferative activities, Bioorg. Med. Chem. Lett., 2015, 25, 389-399.

12 Y. Arun, K. Saranraj, C. Balachandran and P. T. Perumal, Novel spirooxindole-pyrrolidine compounds: synthesis, anticancer and molecular docking studies, Eur. J. Med. Chem., 2014, 74, 50-64.

13 S. M. Rajesh, S. Perumal, J. C. Menéndez, P. Yogeeswari and D. Sriram, Antimycobacterial activity of spirooxindolopyrrolidine, pyrrolizine and pyrrolothiazole hybrids obtained by a three-component regio- and stereoselective 1,3-dipolar cycloaddition, MedChemComm, 2011, 2, 626-630.

14 G. Bhaskar, Y. Arun, C. Balachandran, C. Saikumar and P. T. Perumal, Synthesis of novel spirooxindole derivatives by one pot multicomponent reaction and their antimicrobial activity, Eur. J. Med. Chem., 2012, 51, 79-91.

15 E. Rajanarendar, S. Ramakrishna, K. G. Reddy, D. Nagaraju and Y. N. Reddy, A facile synthesis, anti-inflammatory and analgesic activity of isoxazolyl-2,3-dihydrospiro[benzo[f] isoindole-1,3'-indoline]-2',4,9-triones, Bioorg. Med. Chem. Lett., 2013, 23, 3954-3958.

16 M. J. Kornet and A. P. Thio, Oxindole-3-spiropyrrolidines and -piperidines. Synthesis and local anesthetic activity, J. Med. Chem., 1976, 19, 892-898.

17 N. Arumugam, A. I. Almansour, R. S. Kumar, D. Kotresha, R. Saiswaroop and S. Venketesh, Dispiropyrrolidinylpiperidone embedded indeno[1,2-b]quinoxaline heterocyclic hybrids: Synthesis, cholinesterase inhibitory activity and their molecular docking simulation, Bioorg. Med. Chem., 2019, 27, 2621-2628.

18 Y. Kia, H. Osman, R. S. Kumar, A. Basiri and V. Murugaiyah, Synthesis and discovery of highly functionalized mono- and bis-spiro-pyrrolidines as potent cholinesterase enzyme inhibitors, Bioorg. Med. Chem. Lett., 2014, 24, 1815-1819.

19 Y. Kia, H. Osman, R. S. Kumar, V. Murugaiyah, A. Basiri, S. Perumal and I. A. Razak, A facile chemo-, regio- and stereoselective synthesis and cholinesterase inhibitory activity of spirooxindole-pyrrolizine-piperidine hybrids, Bioorg. Med. Chem. Lett., 2013, 23, 2979-2983.

20 R. Ranjith Kumar, S. Perumal, P. Senthilkumar, P. Yogeeswari and D. Sriram, Discovery of antimycobacterial spiro-piperidin-4-ones: an atom economic, stereoselective synthesis, and biological intervention, J. Med. Chem., 2008, 51, 5731-5735.

21 N. Arumugam, A. I. Almansour, R. S. Kumar, M. Altaf, R. Padmanaban, P. Sureshbabu, G. Angamuthu, D. Kotresha, T. S. Manohar and S. Venketesh,
Spiropyrrolidine/spiroindolizino[6,7-b]indole heterocyclic hybrids: Stereoselective synthesis, cholinesterase inhibitory activity and their molecular docking study, Bioorg. Chem., 2018, 79, 64-71.

22 N. Arumugam, A. I. Almansour, R. S. Kumar, V. S. Periasamy, J. Athinarayanan, A. A. Alshatwi, P. Govindasami, M. Altaf and J. C. Menéndez, Regio- and diastereoselective synthesis of anticancer spirooxindoles derived from tryptophan and histidine via three-component 1,3-dipolar cycloadditions in an ionic liquid, Tetrahedron, 2018, 74, 5358-5366.

23 N. Arumugam, A. I. Almansour, R. S. Kumar, M. Altaf, S. M. Mahalingam, G. Periyasami, J. C. Menéndez and A. J. M. A. Al-Aizari, Multicomponent domino protocol for the stereoselective synthesis of novel pyrrolo[3,2-c] quinolinone hybrid heterocycles, Tetrahedron Lett., 2019, 60, 602-605.

24 A. I. Almansour, N. Arumugam, R. S. Kumar, D. Kotresha, T. S. Manohar and S. Venketesh, Design, synthesis and cholinesterase inhibitory activity of novel spiropyrrolidine tethered imidazole heterocyclic hybrids, Bioorg. Med. Chem. Lett., 2019, 30, 126789.

25 R. S. Kumar, A. I. Almansour, N. Arumugam and R. Ranjith Kumar, Substitution induced switch between PictetSpengler and Eschweiler-Clarke reactions: Selective synthesis of spiro acenaphthylene pyrrolo[1,2-b]isoquinoline/pyrrolidine hybrids, Tetrahedron Lett., 2020, 61, 151606.

26 A. I. Almansour, N. Arumugam, R. S. Kumar, D. Kotresha, T. S. Manohar and S. Venketesh, Design, synthesis and cholinesterase inhibitory activity of novel spiropyrrolidine tethered imidazole heterocyclic hybrids, Bioorg. Med. Chem. Lett., 2019, 30, 126789.

27 A. Y. Barkov, N. S. Zimnitskiy, V. Y. Korotaev, I. B. Kutyashev, V. S. Moshkin and V. Y. Sosnovskikh, Regio- and stereoselective 1,3-dipolar cycloaddition of indenoquinoxalinone azomethine ylides to $\beta$-nitrostyrenes: synthesis of spiro[indeno[1,2-b] quinoxaline-11, $3^{\prime}$ pyrrolizidines] and spiro[indeno[1,2-b] quinoxaline-11,2'pyrrolidines], Chem. Heterocycl. Compd., 2017, 53, 451-459.

28 A. M. Akondi, S. Mekala, M. L. Kantam, R. Trivedi, L. R. Chowhan and A. Das, An expedient microwave assisted regio- and stereoselective synthesis of spiroquinoxaline pyrrolizine derivatives and their AChE inhibitory activity, New J. Chem., 2017, 41, 873-878.

29 Y. Sarrafi, A. Asghari, M. Hamzehloueian, K. Alimohammadi and M. Sadatshahabi, A facile regioselective synthesis of novel spiroacenaphthene pyrroloisoquinolines through 1,3dipolar cycloaddition reactions, J. Braz. Chem. Soc., 2013, 24, 1957-1963.

30 D. E. Worrall, C. S. Marvel and W. H. Lycan, Org. Synth., 1941, 1, 413.

31 A. I. Almansour, N. Arumugam, R. S. Kumar, D. M. Althamili, G. Periyasami, K. Ponmurugan, N. A. Al-Dhabi, K. Perumal and D. Premnath, Domino multicomponent approach for the synthesis of functionalized spiro-Indeno [1,2-b]quinoxaline heterocyclic hybrids and their 
antimicrobial activity, synergistic effect and molecular docking simulation, Molecules, 2019, 24, 1962.

32 S. M. Rajesh, B. D. Bala and S. Perumal, Multi-component, 1,3-dipolar cycloaddition reactions for the chemo-, regioand stereoselective synthesis of novel hybrid spiroheterocycles in ionic liquid, Tetrahedron Lett., 2012, 53, 5367-5371.

33 N. Arumugam, A. I. Almansour, R. S. Kumar, D. M. AlThamili, G. Periyasami, V. S. Periasamy, J. Athinarayanan, A. A. Alshatwi, S. M. Mahalingam and J. C. Menéndez, Regio and stereoselective synthesis of anticancer spirooxindolopyrrolidine embedded piperidone heterocyclic hybrids derived from one-pot cascade protocol, Chem. Cent. J., 2018, 12, 95.

34 R. S. Kumar, A. I. Almansour, N. Arumugam, D. M. Q. Althomili, M. Altaf, A. Basiri, D. Kotresha, T. S. Manohar and S. Venketesh, Ionic liquid-enabled synthesis, cholinesterase inhibitory activity, and molecular docking study of highly functionalized tetrasubstituted pyrrolidines, Bioorg. Chem., 2018, 77, 263-268.

35 Crystalographic data (including structure factors) for the compounds $6 \mathbf{e}$ in this article have been deposited with the Cambridge Crystallographic Data Centre as supplementary publication number CCDC 1966793. Copies of the data can be obtained, free of charge, on application to CCDC, 12 Union Road, Cambridge CB2 1EZ, UK [fax: +44 (0)1223 336033 or e-mail: ]deposit@ccdc.cam.ac.uk.
36 Stoe \& Cie, $X$-AREA and $X$-RED32, Stoe \& Cie, Darmstadt, Germany, 2002.

37 G. M. Sheldrick, SHELXT - Integrated space-group and crystal-structure determination, Acta Crystallogr., Sect. A: Found. Crystallogr., 2013, 71, 3-8.

38 G. M. Sheldrick, Crystal structure refinement with SHELXL, Acta Crystallogr., Sect. C: Struct. Chem., 2015, 71, 3-8.

39 L. J. Farrugia, WinGX suite for small-molecule single-crystal crystallography, J. Appl. Crystallogr., 1999, 32, 837-838.

40 A. L. Spek, Structure validation in chemical crystallography, Acta Crystallogr., Sect. D: Biol. Crystallogr., 2009, 65, 148-155.

41 S. Kansiz, N. Dege, A. S. Aydin, E. Ağar and I. P. Matushko, Crystal structure and Hirshfeld surface analysis of (Z)-6-[(2hydroxy-5-nitroanilino)methylidene]-4-methylcyclohexa-2,4dien-1-one, Acta Crystallogr., Sect. E: Crystallogr. Commun., 2019, 75, 812-815.

42 M. Boulhaoua, S. Kansiz, M. El Hafi, S. Lahmidi, N. Dege, M. Benchidmi and J. T. Mague, Crystal structure and Hirshfeld surface analysis of 5-[(5-nitro-1H-indazol-1-yl) methyl]-3-phenyl-4,5-dihydroisoxazole, Acta Crystallogr., Sect. E: Crystallogr. Commun., 2019, 75, 71-74.

43 A. Qadir, S. Kansiz, G. M. Rosair, N. Dege and T. S. Iskenderov, Crystal structure and Hirshfeld surface analysis of a copper(II) complex with ethylenediamine and non-coordinated benzoate, Acta Crystallogr., Sect. E: Crystallogr. Commun., 2020, 76, 111-114. 